\title{
Validación de la escala para manía de la Universidad Nacional de Colombia usando el análisis de Rasch
}

\author{
Ricardo Sánchez, Juliana Velásquez, Álvaro Navarro \\ Facultad de Medicina, Universidad Nacional de Colombia, Bogotá, D.C., Colombia
}

Introducción. La escala para manías de la Universidad Nacional de Colombia es un instrumento diseñado para medir la gravedad de la sintomatología maníaca y sólo ha sido validada utilizando técnicas de teoría psicométrica clásica.

Objetivos. Determinar las propiedades psicométricas y las características, como instrumento de medición, de la escala para manías mediante análisis basados en la teoría de respuesta al ítem.

Materiales y métodos. Se evaluaron 264 pacientes con diagnóstico de episodio maníaco, hipomaníaco o mixto, usando la escala para manías. Se analizaron las características psicométricas del instrumento utilizando un modelo de Rasch de crédito parcial para datos politómicos.

Resultados. El análisis mostró que, en contraste con los ítems, los índices de separación y la confiabilidad para personas fueron bajos, lo cual sugiere una posible representación reducida del constructo evaluado en esta muestra. La disminución de la necesidad de dormir fue el ítem más fácilmente detectable en los pacientes maníacos. La mayoría de ítems se ajustó a las expectativas del modelo (excepto el afecto depresivo y la capacidad de distraerse). El diagnóstico de la escala de puntuación mostró que las medidas promedio se incrementan monotónicamente a través de la escala. Dos ítems mostraron redundancia y podrían omitirse en versiones futuras de la escala. El mapa persona-ítem sugiere que el síndrome no queda completamente evaluado por la escala, probablemente debido a que algunos síntomas depresivos no se incluyeron en ella.

Conclusiones. En este primer estudio de la escala para manías usando el análisis de Rasch, se detectó mal ajuste y redundancia de algunos ítems. El síndrome maníaco no queda completamente evaluado por la escala. El instrumento podría mejorarse agregando síntomas depresivos.

Palabras clave: escalas de valoración psiquiátrica, estudios de validación, trastorno bipolar/diagnóstico, psicometría/estadísticas y datos numéricos, reproducibilidad de resultados, entrevista psicológica.

Validation by Rasch analysis of the Mania Scale (EMUN) for measuring manic symptions

Introduction. The Mania Scale (EMUN) developed at the Universidad Nacional de Colombia was designed to measure the severity of manic symptoms, but has been validated only using classical psychometric theory.

Objectives. The psychometric properties and measuring characteristics of the EMUN scale were determined using an analysis based on item response theory.

Materials and methods. Two hundred sixty-four patients with manic, hypomanic or mixed episode symptoms were assessed using the EMUN scale. The psychometric characteristics of the scale were analyzed using a Rasch model for partial credit scoring.

Results. The analysis based on the item response theory showed that reliability and separation indexes for persons are low in contrast to items. This suggested a narrow representation of the construct evaluated in this sample. Reduced need to sleep has been the most easily detectable symptom in mania. Excepting depressive affect and distractibility, the majority of items fit the model's expectation The rating scale diagnostics showed that the average measures increase monotonically across the rating scale. Two items showed redundancy and can be omitted in future versions of the scale. The person-item map suggested that the syndrome is not fully evaluated by the scale, probably because some depressive symptoms are not included.

Conclusion. In this first study to use Rasch analysis to assess the psychometric properties of the EMUN scale, misfit and redundancy of items have been detected. The manic syndrome is not fully evaluated by the scale. The instrument can be improved by adding depressive symptoms.

Key words: Psychiatric status rating scales, validation studies, bipolar disorder/diagnosis, psychometrics/statistics and numerical data, reproducibility of results; interview, psychological. 
El trastorno afectivo bipolar es una enfermedad grave, difícilmente curable y recurrente, con síntomas persistentes por largos periodos, en la mayoría de los casos. Este tipo de trastorno se considera una enfermedad crónica con una importante repercusión sobre la calidad de vida, que genera altos niveles de discapacidad (1-3) y mayor riesgo de mortalidad (4).

En cuanto a su prevalencia, se estima en 1,2\% para el de tipo I (episodios maníacos o mixtos), con mayor frecuencia en hombres, y para el de tipo II (episodios hipomaníacos, únicamente), la prevalencia es 0,5 $\%$. Para el episodio maniaco, la prevalencia se ha calculado en 2,1 \% para hombres y 1,5\% para mujeres. La prevalencia estimada de cualquier trastorno afectivo en la población colombiana es $11,7 \%$ para hombres y $17,5 \%$ para mujeres (5).

Algunos estudios han reportado que un adecuado diagnóstico del trastorno afectivo bipolarusualmente lleva entre 8 y 10 años, que un mal diagnóstico lleva a errores en la formulación de tratamientos que pueden generar un mal pronóstico y que el uso de antidepresivos produce virajes a manías o puede deteriorar el curso y el pronóstico de la enfermedad (6-12).

Teniendo en cuenta que el diagnóstico de esta entidad es una tarea fundamentalmente clínica, se han desarrollado múltiples instrumentos para hacer más válido y objetivo el resultado de la evaluación. Las escalas para manía comenzaron a desarrollarse a principios de los años 70 . Las primeras eran extensas y complejas, con tiempos de aplicación prolongados y necesidad de entrenamiento especial de los evaluadores que las aplicaban. Con el tiempo, se han desarrollado herramientas de medición cortas y de más fácil utilización. Además, se ha ampliado el espectro de síntomas que cubren, pasando de medir solamente síntomas de manía pura o clásica a la inclusión de síntomas descritos en cuadros disfóricos y episodios mixtos (13). Por otra parte, se han desarrollado instrumentos de autorreporte, que son calificados directamente por el paciente $(14,15)$. También, se han generado instrumentos cortos que agilizan el proceso de evaluación de los pacientes y llegan, incluso, al desarrollo de escalas de sólo 10 ítems $(16,17)$.

Correspondencia:

Ricardo Sánchez, Carrera 68 № 24-B-51, apartamento 612, Bogotá, D.C., Colombia

rsanchezpe@unal.edu.co

Recibido:15/12/10; aceptado:14/06/11
Aunque se han efectuado validaciones de instrumentos para ser utilizados en pacientes que hablen idiomas diferentes del inglés (18), hasta donde tenemos conocimiento, el único instrumento original para medir síntomas maníacos que se ha desarrollado en poblaciones hispanoparlantes es la escala para manía de la Universidad Nacional de Colombia (19); es una escala para ser aplicada por clínicos con experiencia en evaluación de pacientes psiquiátricos, que cuenta con 16 ítems, que cubren los siguientes cuatro dominios del síndrome maníaco:

Activación psíquica: conformado por síntomas como urgencia del pensamiento, fuga de ideas, capacidad de distraerse, ánimo emprendedor y afecto eufórico.

Activación física: conformado por síntomas como hiperactividad, aumento del contacto interpersonal, aumento de la intensidad de la voz y afecto irritable.

Activación autonómica y sus efectos: los síntomas que integran este dominio son la disminución de la necesidad de dormir, la hipersexualidad, el exceso de energía y las alteraciones en el sueño.

Depresión-megalomanía: conformado por síntomas como afecto depresivo, ideas depresivas y de grandiosidad.

Se ha reportado que la escala para manías es un instrumento válido y confiable para medir el síndrome maníaco (19). En el estudio que hizo su validación inicial se reportó que la coherencia interna para cada uno de los dominios fue buena (valores de alfa de Cronbach mayores de 0,7) y los coeficientes de confiabilidad fueron mayores de 0,9; el instrumento también demostró capacidad para detectar cambios en la condición clínica luego del tratamiento (19).

Las anteriores características relacionadas con validez, confiabilidad y sensibilidad al cambio, se derivaron de la utilización de métodos de la teoría clásica de los test. Sin embargo, tiene la limitación de no poder estimar la dificultad de un ítem de manera independiente de las características del rasgo que se está midiendo en un paciente (20). Además, sólo considera un único estimador de confiabilidad (junto con su correspondiente error estándar), sin poder incorporar el hecho de que la precisión de las mediciones varía según el nivel de intensidad del atributo. La teoría de respuesta al ítem permite modelar la asociación entre el nivel que tiene un paciente en un determinado rasgo 
(variable latente) y la probabilidad de responder a un ítem, mediante el uso de un modelo que incorpora una función monotónica no lineal (21).

Entre los modelos basados en la teoría de respuesta al ítem, el modelo de Rasch, o modelo de un parámetro, ha ganado popularidad en el área de la validación de escalas en salud, teniendo en cuenta que permite, no sólo medir el ajuste entre el modelo y la muestra de pacientes, sino también, evaluar las características de los métodos de medición incorporados en el instrumento (sistema o escala de medición de los ítems). En este modelo, la probabilidad de que una persona responda a un ítem es una función logística de la diferencia entre la habilidad de la persona y la dificultad del ítem (22).

Como objetivo de este estudio se planteó determinar las propiedades psicométricas de la escala para manía, utilizando una metodología de análisis basada en la teoría de respuesta al ítem.

\section{Material y métodos}

Diseño. Se diseñó un estudio transversal de tipo psicométrico.

Pacientes. Se evaluaron 264 pacientes con diagnóstico de episodio maníaco, hipomaníaco o mixto, que asistieron a los servicios de urgencias de la Clínica Nuestra Señora de la Paz en Bogotá, durante los años 2009 y 2010. Para incluir a un paciente en el estudio, se requería que alguno de los diagnósticos anteriores hubiera sido hecho por dos psiquiatras clínicos, generalmente pertenecientes a los servicios de urgencias y hospitalización, usando el sistema DSM-IV. Solamente se incluyeron los pacientes que se valoraron con la escala dentro de los tres primeros días luego de su ingreso.

Procedimientos. La escala se aplicó en el contexto de una entrevista clínica semiestructurada efectuada dentro de los ejercicios académicos de los estudiantes de posgrado de la Universidad Nacional de Colombia, por un clínico con experiencia en el uso del instrumento. La calificación de la escala se efectuaba luego de terminar la entrevista del paciente. Además, se registraban datos provenientes de la historia clínica del paciente, como la edad, y aspectos relacionados con la evolución de la enfermedad y el tipo de tratamiento.

La realización del estudio supuso recolectar la información anterior que quedaba consignada en la historia clínica y fue aprobado por el Comité de Ética de la Clínica Nuestra Señora de la Paz.
Instrumentos. Se utilizó un formato para consignar los resultados de las mediciones sobre variables demográficas y clínicas. Además, se aplicó la escala para manía.

Análisis estadístico. Se utilizaron técnicas de estadística descriptiva para resumir las características de las variables demográficas y clínicas. El comportamiento de los puntajes dentro de los diferentes ítems y dominios se describió mediante el uso de medianas y sus correspondientes rangos intercuartílicos como medidas de dispersión.

Considerando que cada uno de los ítems fue medido con una escala ordinal, se utilizó un modelo de Rasch de crédito parcial para datos politómicos (23). En el análisis de Rasch se consideraron tres aspectos:

Evaluación de la confiabilidad de ítems y personas. Para esto se calcularon los índices de confiabilidad de ítems y personas. Además, se estimaron los índices de separación de ítems y personas. Como criterio de corte, se consideró que los valores mayores de dos eran buenos indicadores de separación (22).

Análisis de ajuste de los ítems. Para ubicar los ítems que no se ajustaban al modelo, se calcularon los puntajes de cuadrados medios, teniendo en cuenta los valores de ajuste interno y externo (infit y outfit) entre 0,6 y 1,4 . Se considera que existe mal ajuste de un ítem cuando tiene valores de ajuste mayores de 1,4 junto con puntajes mayores de 2 en la estandarización z. Se declaran redundantes los ítems con valores de ajuste menores de 0,6 (24). Los valores de cuadrados medios del ajuste externo sirven para evaluar el ajuste de los datos al modelo, mientras que los valores de cuadrados medios del ajuste interno permiten evaluar el ajuste de los ítems dentro del síndrome. Con estos datos es posible calcular tanto la homogeneidad del constructo (síndrome maníaco) como la redundancia de los ítems (22). Además, se utilizó el mapa personas-ítems.

Diagnóstico del sistema de calificación de la escala. Para esto se calcularon las medidas promedio sobre escala logit, cuadrados medios de ajuste interno y externo y medidas de step (25).

Para el estudio se tuvo en cuenta la recomendación de que el tamaño de muestra para este tipo de análisis sea de, al menos, 250 observaciones $(20,26)$. Los análisis estadísticos descriptivos se 
hicieron con el programa $\mathrm{R}$ y el análisis de Rasch se efectuó con el programa Winsteps ${ }^{\circledR}$.

\section{Resultados}

El total de pacientes evaluados tuvo una media de edad de 37 años (de 13 años). El $54 \%$ de los pacientes de la muestra eran mujeres. En relación con el uso de medicamentos, $25 \%$ de los pacientes $(n=66)$ recibían antipsicóticos, 26,5 $\%$ tomaban carbonato de litio $(n=70), 39,8 \%$ recibían anticonvulsivos $(n=105)$ y $32,6 \%$ usaban benzodiacepinas $(n=86)$. La distribución según el diagnóstico se muestra en el cuadro 1.

En relación con la calificación dada a cada uno de los ítems del instrumento, la mediana más alta la tuvo el ítem que midió la disminución de la necesidad de dormir (mediana $=4$, rango intercuartílico=1), mientras que las medianas más bajas fueron las de los ítems que miden afecto e ideas depresivas (mediana de cero en los dos ítems, rango intercuartílico de 2 y 1 , respectivamente).

Cuadro 1. Distribución de los pacientes según diagnóstico

\begin{tabular}{lrc}
\hline Tipo de episodio & \multicolumn{1}{c}{$\mathbf{n}$} & \multicolumn{1}{c}{$\%$} \\
\hline Hipomaníaco & 11 & 4,17 \\
Maníaco grave & 191 & 72,35 \\
Maníaco leve o moderado & 53 & 20,08 \\
Mixto grave & 5 & 1,89 \\
Mixto leve o moderado & 4 & 1,52 \\
Total & 264 & 100 \\
\hline
\end{tabular}

En el cuadro 2 se presenta la información relacionada con el ajuste del modelo de Rasch. Estos datos sugieren un inadecuado ajuste para los ítems (la desviación estándar del ajuste interno y externo para los ítems en ZSTD es mayor de 2). Además, se evidencian valores de separación menores de dos para las personas.

En el cuadro 3 se presentan los índices de confiabilidad y separación para personas e ítems correspondientes a los cuatro dominios. La confiabilidad muestra valores bajos de correlación para personas (incluso, hay valores cercanos a 0,2 en uno de los dominios) y mayor de 0,94 para ítems. En relación con los índices de separación, se encontraron valores bajos para personas pero buenos para los ítems. Este hallazgo (bajos índices de separación y confiabilidad para personas, en contraste con altos índices para los ítems) apunta a que la muestra utilizada tiene restricción en el rango del constructo que se está midiendo.

En el cuadro 4 se presentan los valores de los datos estadísticos de ajuste interno y externo de los ítems de la escala. El análisis de Rasch insinúa mal ajuste de los ítems que miden depresión y capacidad de distraerse, y sugiere redundancia de los que miden urgencia del pensamiento y aumento de energía.

En el cuadro 5 se muestran las medidas promedio (en unidades logito) y los cuadrados medios de ajuste interno y externo. Los valores

Cuadro 2. Información global sobre el ajuste del modelo para la escala para manías

\begin{tabular}{|c|c|c|c|c|c|c|c|}
\hline & & \multicolumn{2}{|c|}{ Ajuste interno } & \multicolumn{2}{|c|}{ Ajuste externo } & \multirow[t]{2}{*}{ Separación } & \multirow[t]{2}{*}{ Confiabilidad } \\
\hline & & $\bar{X}$ al cuadrado & z estándar & $\bar{X}$ al cuadrado & z estándar & & \\
\hline Personas & $\begin{array}{c}\text { Media } \\
\text { DE }\end{array}$ & $\begin{array}{l}1,00 \\
0,56\end{array}$ & $\begin{array}{r}-0,1 \\
1,5\end{array}$ & $\begin{array}{l}1,07 \\
0,69\end{array}$ & $\begin{array}{l}0,0 \\
1,5\end{array}$ & 1,60 & 0,72 \\
\hline Ítems & $\begin{array}{c}\text { Media } \\
\text { DE }\end{array}$ & $\begin{array}{l}1,01 \\
0,31\end{array}$ & $\begin{array}{r}-0,2 \\
3,5\end{array}$ & $\begin{array}{l}1,07 \\
0,41\end{array}$ & $\begin{array}{l}0,0 \\
3,8\end{array}$ & 9,17 & 0,99 \\
\hline
\end{tabular}

DE: desviación estándar

Cuadro 3. Índices de confiabilidad y separación para las subescalas de la escala para manías

\begin{tabular}{lcccccccc}
\hline & \multicolumn{2}{c}{$\begin{array}{c}\text { Dominio 1: } \\
\text { Activación } \\
\text { psíquica }\end{array}$} & \multicolumn{2}{c}{$\begin{array}{c}\text { Dominio 2: } \\
\text { Activación } \\
\text { física }\end{array}$} & & \multicolumn{2}{c}{$\begin{array}{c}\text { Dominio 3: } \\
\text { Activación } \\
\text { autonómica y } \\
\text { sus efectos }\end{array}$} & \multicolumn{2}{c}{$\begin{array}{c}\text { Dominio 4: } \\
\text { Polaridad } \\
\text { depresión } \\
\text { megalomanía }\end{array}$} \\
& Persona & Ítem & Persona & Ítem & Persona & Ítem & Persona & Ítem \\
\hline Índice de confiabilidad & 0,61 & 0,98 & 0,69 & 0,94 & 0,60 & 0,99 & 0,23 & 0,99 \\
Índice de separación & 1,25 & 7,49 & 1,48 & 4,07 & 1,22 & 9,28 & 0,54 & 11,95 \\
\hline
\end{tabular}


correspondientes a las medidas promedio se incrementan de forma monotónica y de la manera esperada, mediante los puntajes de la escala, lo cual sugiriere que los pacientes con mayor intensidad del atributo (manías más graves) puntúan más alto en los ítems. Este hallazgo sugiere una estructura adecuada del sistema de calificación usado en cada ítem, aunque no descarta redundancia.

Los valores de cuadrados medios de ajuste interno y externo se encuentran en un rango entre 0,65 y 2,1 . La mayoría de los valores de cuadrados medios de ajuste interno y externo fueron cercanos a uno; sin embargo, para el nivel de puntuación dos del dominio tres y cuatro del dominio cuatro, los datos sugieren que los pacientes con altos niveles de manía impredeciblemente son calificados con bajos puntajes en el dominio tres y que los pacientes con niveles bajos de manía son calificados con puntajes altos en el dominio cuatro.

En el mapa personas-ítems (figura 1), entre más arriba esté posicionado un paciente en el eje vertical, más intensidad tendrá en la manía. Aunque todos los ítems quedaron incorporados dentro del rango de dispersión de los puntajes obtenidos por los pacientes, el mapa muestra una restricción en el rango de respuesta, ya que los pacientes se distribuyen en un rango estrecho, entre -1 y 1 logito. Tanto la media de las personas como la de los ítems, se encuentran próximas una de otra, cercanas al valor de cero logitos, lo que sugiere que el valor medio del constructo que mide esta escala es cuantitativamente similar al puntaje medio que presentan los pacientes de la muestra estudiada. La posición en la zona superior de la escala del ítem que mide disminución de necesidad de dormir, indica que este síntoma es fácil de ubicar como marcador de manía. Esto contrasta con el ítem que mide ideas depresivas, el cual se muestra como un síntoma que no se presenta con frecuencia en el constructo. Otra información importante que aporta el mapa es que hay un espacio descubierto de más de medio logito entre síntomas como hiperbuliasexualidad, y síntomas depresivos (en este punto del mapa se observan varios signos \#\#\#, que corresponden a personas sin un ítem o conjunto de ítems que estén midiendo algún punto del atributo) (figura 1).

\section{Discusión}

La escala para manía es, hasta donde tenemos conocimiento, el único instrumento desarrollado en una población de pacientes hispanoparlantes para medir el síndrome maniaco. Con su desarrollo, se trató de ensamblar un instrumento sensible, que incorporara la mayor cantidad de características clínicas del síndrome, para lo cual se tuvo en cuenta, no sólo la información proveniente de las escalas disponibles hasta la fecha, sino datos provenientes de la experiencia de los pacientes y de expertos clínicos. En su construcción se trató de ensamblar un instrumento con una estructura que facilitara su aplicación y permitiera una medición más confiable; para esto se incorporaron ejemplos

Cuadro 4. Estadísticas de ajuste de los ítems para la escala para manías

Estadísticas de ajuste de los ítems

\begin{tabular}{lcccc} 
Ítem & $\bar{X}$ interna al cuadrado & $\mathbf{z}$ interna estándar & $\bar{X}$ externa al cuadrado & z externa estándar \\
\hline Euforia (1) & 1,22 & 2,3 & 1,26 & 2,6 \\
Irritabilidad (2) & 0,96 & $-0,5$ & 0,96 & $-0,4$ \\
Depresión (4) & 1,62 & 5,5 & 2,12 & 6,9 \\
Urgencia del pensamiento (1) & 0,51 & $-6,1$ & 0,51 & $-6,3$ \\
Fuga de ideas (1) & 1,09 & 0,9 & 1,10 & 1,0 \\
Intensidad de la voz (2) & 0,67 & $-4,6$ & 0,66 & $-4,6$ \\
Ideas de grandiosidad (4) & 0,94 & $-0,8$ & 0,93 & $-0,9$ \\
Ideas depresivas (4) & 1,18 & 1,7 & 1,46 & 0,9 \\
Hiperactividad (2) & 1,07 & 0,8 & 1,08 & $-6,1$ \\
Exceso de energía (3) & 0,54 & $-6,0$ & 0,53 & $-2,8$ \\
Hiperbulia (1) & 0,78 & $-3,0$ & 0,78 & 2,4 \\
Disminución del sueño (3) & 1,26 & 2,5 & 1,24 & 3,4 \\
Hipersexualidad (3) & 1,24 & 3,0 & 1,52 & 4,9 \\
Capacidad de distraerse (1) & 1,44 & 4,4 & 1,01 & 0,2 \\
Aumento de sociabilidad (2) & 1,04 & 0,5 & 0,66 & $-4,4$ \\
Alteraciones del juicio (3) & 0,68 & $-4,4$ & & \\
\hline
\end{tabular}

Entre paréntesis se indica el dominio al cual se adjudica cada ítem en la validación original. 
Cuadro 5. Mediciones de las categorías para la escala para manías

\begin{tabular}{|c|c|c|c|}
\hline Dominio y categoría & $\begin{array}{c}\text { Medida } \\
\text { promedio }\end{array}$ & $\begin{array}{l}\bar{X} \text { al cuadrado, } \\
\text { ajuste interno }\end{array}$ & $\begin{array}{l}\bar{X} \text { al cuadrado, } \\
\text { ajuste externo }\end{array}$ \\
\hline \multicolumn{4}{|c|}{ Dominio 1. Activación psíquica } \\
\hline 0 & $-0,74$ & 1,18 & 1,27 \\
\hline 1 & $-0,67$ & 0,97 & 0,98 \\
\hline 2 & $-0,41$ & 0,93 & 0,92 \\
\hline 3 & $-0,01$ & 0,88 & 0,89 \\
\hline 4 & 0,32 & 0,98 & 0,99 \\
\hline 5 & 0,64 & 0,99 & 0,99 \\
\hline \multicolumn{4}{|c|}{ Dominio 2. Activación física } \\
\hline 0 & $-0,59$ & 1,17 & 1,21 \\
\hline 1 & $-0,55$ & 0,87 & 0,84 \\
\hline 2 & $-0,33$ & 0,80 & 0,78 \\
\hline 3 & 0,01 & 0,90 & 0,89 \\
\hline 4 & 0,41 & 0,79 & 0,81 \\
\hline 5 & 0,66 & 0,83 & 0,86 \\
\hline \multicolumn{4}{|c|}{ Dominio 3. Activación autonómica y sus efectos } \\
\hline 0 & $-0,77$ & 1,10 & 1,17 \\
\hline 1 & $-0,65$ & 0,79 & 0,77 \\
\hline 2 & $-0,44$ & 0,69 & 0,65 \\
\hline 3 & 0,04 & 0,87 & 0,87 \\
\hline 4 & 0,44 & 0,83 & 0,85 \\
\hline 5 & 0,76 & 0,92 & 0,95 \\
\hline \multicolumn{4}{|c|}{ Dominio 4. Polaridad depresión-megalomanía } \\
\hline 0 & $-1,43$ & 1,17 & 1,12 \\
\hline 1 & $-1,22$ & 1,18 & 1,31 \\
\hline 2 & $-0,82$ & 1,24 & 1,67 \\
\hline 3 & $-0,31$ & 1,26 & 1,69 \\
\hline 4 & 0,17 & 1,21 & 2,18 \\
\hline 5 & 0,39 & 1,10 & 1,23 \\
\hline
\end{tabular}

El puntaje va desde cero (ausente) hasta cinco (lo máximo clínicamente observable)

de preguntas "tipo", en los casos en los cuales la exploración del síntoma requiriera este tipo de abordaje durante la entrevista, y se generó una escala de calificación de ítems con descripciones que permitieran una calificación más precisa de la sintomatología (19). En la validación inicial de este instrumento, se utilizaron métodos psicométricos de la teoría clásica de los test.

El presente estudio propone una validación mediante una estrategia de la teoría de respuesta al ítem (análisis de Rasch), ya que se han reportado ventajas con esta aproximación, teniendo en cuenta la posibilidad de obtener información de los estimadores que es independiente de las características de la muestra estudiada; esta propiedad, también conocida como "invarianza", es una de las fortalezas de esta metodología en comparación con los enfoques clásicos $(21,27)$.

Las características de la muestra empleada indican que el espectro de la enfermedad que presentaban los pacientes estudiados no fue suficientemente amplio: en estudios posteriores se recomienda incorporar una mayor cantidad de pacientes con cuadros menos graves o pacientes que presenten cuadros mixtos o incluso de depresión bipolar.

En cuanto a la calificación de los síntomas, nuestros hallazgos coinciden con los de la mayoría de estudios que reportan que las alteraciones del sueño son el síntoma más frecuente en episodios maníacos (28); por otro lado, la baja calificación que se encontró en el grupo de síntomas depresivos, puede relacionarse con la baja frecuencia de pacientes con cuadros mixtos, en los cuales la depresión es un hallazgo más frecuente (29). Encontramos deficiencias en el ajuste de algunos ítems de la escala ("ideas depresivas" y "capacidad de distraerse") que no se insinuaban en el estudio inicial de validación del instrumento.

En cuanto al ítem que mide el afecto depresivo, consideramos que la forma en que se explora esta característica clínica puede ser responsable del mal ajuste: mientras que los dos ítems previos que en la escala para manías miden afecto (euforia o irritabilidad) están presentados como 


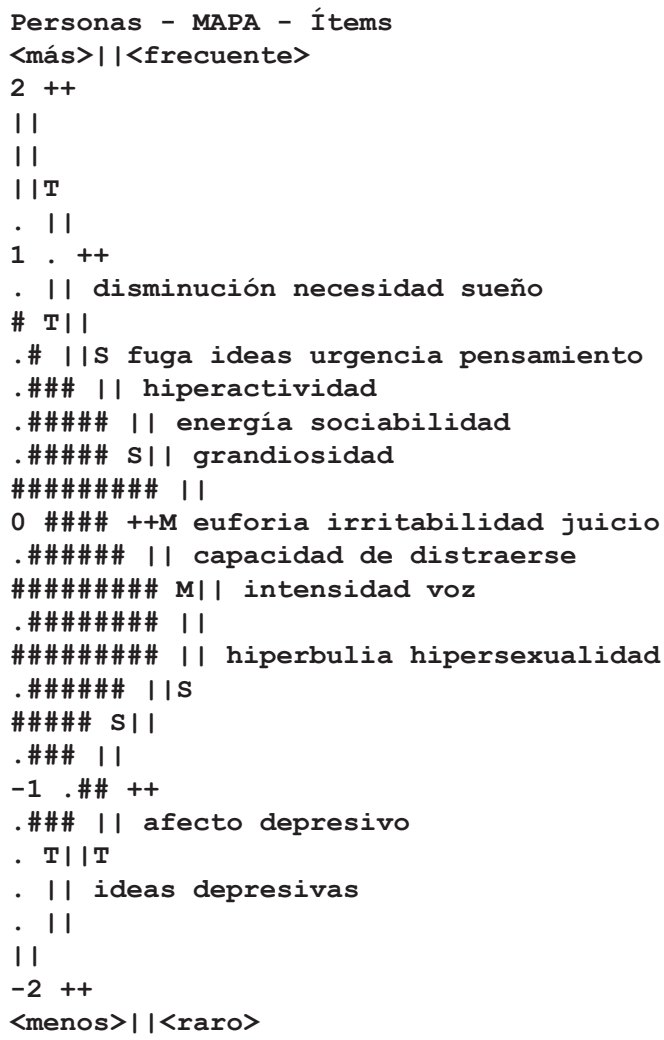

Al lado izquierdo, cada '\#' son tres personas y un punto indica una persona. Las personas están ubicadas sobre una escala que mide la intensidad del atributo. Al lado derecho, se presentan los ítems ubicados sobre la escala de dificultad. Se utiliza una escala en unidades logit, tanto para personas como para ítems.

Figura 1. Mapa de personas-ítems

"presencia de uno o más períodos claramente diferentes del estado basal, que duran más de una hora, en los cuales el paciente está muy alegre o irritable", el afecto depresivo se explora de una manera más inespecífica ("sentimientos de tristeza, aburrimiento, desgano, incapacidad de disfrutar, pesimismo"). Esta estructura es claramente diferente de las otras diseñadas para explorar afecto. Además, en un solo ítem se están explorando varias condiciones diferentes: tristeza, disforia, anhedonia y desesperanza. Este hallazgo podría relacionarse con la existencia de un espacio descubierto entre los síntomas depresivos y otros síntomas que miden el síndrome (figura 1).

Podría plantearse que una manera de mejorar el problema de ajuste encontrado es generar varios ítems que permitan una exploración más específica de la sintomatología depresiva y que permitan llenar el espacio descubierto que evidencia el mapa personas-ítems. En relación con el ítem que mide la capacidad de distraerse, la razón de su mal ajuste no es fácilmente determinable. Suponemos que en la forma en que está planteado el ítem en la escala ("el paciente fácilmente se distrae con estímulos externos sin importancia"), la medición puede verse fácilmente afectada por las situaciones ambientales; por ejemplo, si el paciente se evalúa en un ambiente con pocos estímulos, la puntuación puede ser baja, así el paciente tenga un nivel alto en el atributo. En el presente estudio no se efectuó una estandarización de las características ambientales en las que se aplicaba la entrevista, por lo cual pudo haberse generado una medición errónea del rasgo que genera el mal ajuste detectado en el ítem. Por lo anterior, recomendamos que en este ítem se incluya información que permita la medición de la atención del paciente bajo condiciones uniformes. El análisis sugiere que el sistema de calificación empleado para medir cada uno de los síntomas es adecuado.

Resultó llamativo que los pacientes con bajos niveles de manía tendieran a calificarse con puntajes altos en el dominio cuatro (polaridad depresión-megalomanía). Esto podría deberse a que los síntomas de activación presentes en formas graves tienden a opacar los síntomas de 
tipo depresivo. Por otro lado, el hecho de que los pacientes con altos niveles del atributo tendieran a calificarse con menor puntaje en el dominio tres (activación autonómica y sus efectos), podría relacionarse con el tratamiento farmacológico que reciben los pacientes con formas graves y que puede enmascarar varios de los síntomas de este dominio.

Otro hallazgo del análisis es la existencia de redundancia por parte de los ítems que miden urgencia del pensamiento y aumento de energía. Como lo muestra el mapa personas-ítems, estos síntomas están en el mismo nivel que la fuga de ideas y de la sociabilidad. El hallazgo de redundancia tiene sentido desde el punto de vista clínico y podría plantearse que una versión posterior de este instrumento omitiera la exploración de estos síntomas. El análisis también evidenció que en el síndrome hay espacios descubiertos que la escala no está evaluando y que, probablemente, corresponden a sintomatología de tipo depresivo. Este hallazgo concuerda con otros estudios que reportan una coexistencia importante de síntomas maníacos y depresivos (30-32), lo cual no se ha incorporado suficientemente en las escalas de medición de síndromes afectivos, los cuales siguen manejando el esquema de polaridad manía-depresión como entidades opuestas e independientes.

En conclusión, en este primer estudio de la escala para manías mediante el análisis de Rasch, se detectó mal ajuste y redundancia de algunos ítems. Se encontró que el síndrome maníaco no queda completamente evaluado por la escala y que el instrumento podría mejorarse agregando síntomas correspondientes al síndrome depresivo.

\section{Conflicto de intereses}

Los autores del presente manuscrito declaramos no tener conflictos de interés relacionados con el contenido del material que estamos sometiendo.

\section{Financiación}

Universidad Nacional de Colombia.

\section{Referencias}

1. Gutiérrez-Rojas L, Gurpegui M, Ayuso-Mateos JL, Gutiérrez-Ariza JA, Ruiz-Veguilla M, Jurado D. Quality of life in bipolar disorder patients: A comparison with a general population sample. Bipolar Disord. 2008;10:625-34.

2. Gutiérrez-Rojas L, Jurado D, Gurpegui M. Factors associated with work, social life and family life disability in bipolar disorder patients. Psychiatry Res. 2011;186:254-60.
3. Ketter TA. Diagnostic features, prevalence, and impact of bipolar disorder. J Clin Psychiatry. 2010;71:e14.

4. Goodwin FK, Fireman B, Simon GE, Hunkeler EM, Lee J, Revicki D. Suicide risk in bipolar disorder during treatment with lithium and divalproex. JAMA. 2003;290:1467-73.

5. MinisteriodelaProtecciónSocial,FundaciónFESSocial. Estudio Nacional de Salud Mental en Colombia. Bogotá, 2003. Fecha de consulta: 20 de octubre de 2010. Disponible en: http://mps.minproteccionsocial.gov.co/vbecontent/ library/documents/DocNewsNo14822DocumentNo1466. pdf.

6. López J, Baca E, Botillo C, Quintero J, Navarro R, Negueruela M, et al. Diagnostic errors and temporal stability in bipolar disorder. Actas Esp Psiquiatr. 2008;36:205-9.

7. Tiller JW, Schweitzer I. Bipolar disorder: Diagnostic issues. Med J Aust. 2010;193(Suppl.):S5-9.

8. Young AH. Bipolar disorder: Diagnostic conundrums and associated comorbidities. J Clin Psychiatry. 2009;70:e26.

9. Moniwa E, Lee TW, Lofchy J. Revisiting the diagnostic challenges of secondary mania and bipolar disorder in a patient with borderline hyperthyroidism. Can J Psychiatry. 2004;49:863-4.

10. Grunze HC. Switching, induction of rapid cycling, and increased suicidality with antidepressants in bipolar patients: Fact or overinterpretation? CNS Spectr. 2008;13:790-5.

11. Geller B, Fox LW, Fletcher M. Effect of tricyclic antidepressants on switching to mania and on the onset of bipolarity in depressed 6- to 12-year-olds. J Am Acad Child Adolesc Psychiatry. 1993;32:43-50.

12. Bauer MS, Wisniewski SR, Marangell LB, Chessick CA, Allen MH, Dennehy EB, et al. Are antidepressants associated with new-onset suicidality in bipolar disorder? A prospective study of participants in the Systematic Treatment Enhancement Program for Bipolar Disorder (STEP-BD). J Clin Psychiatry. 2006;67:48-55.

13. Sánchez R, Rosero N. Revision crítica de escalas de medición de manía. Avances en Medición. 2003;1:37-70.

14. Shugar G, Schertzer S, Toner BB, Di Gasbarro I. Development, use, and factor analysis of a self-report inventory for mania. Compr Psychiatry. 1992;33:325-31.

15. Braunig P, Shugar G, Kruger S. An investigation of the Self-Report Manic Inventory as a diagnostic and severity scale for mania. Compr Psychiatry. 1996;37:52-5.

16. Bernstein IH, Rush AJ, Suppes T, Trivedi MH, Woo A, Kyutoku Y, et al. A psychometric evaluation of the clinicianrated Quick Inventory of Depressive Symptomatology (QIDS-C16) in patients with bipolar disorder. Int J Methods Psychiatr Res. 2009;18:138-46.

17. Dennehy EB, Suppes T, Crismon ML, Toprac M, Carmody TJ, Rush AJ. Development of the Brief Bipolar Disorder Symptom Scale for patients with bipolar disorder. Psychiatry Res. 2004;127:137-45.

18. Ruggero CJ, Johnson SL, Cuellar AK. Spanish-language measures of mania and depression. Psychol Assess. 2004;16:381-5.

19. Sánchez R, Jaramillo L, Gómez C. Desarrollo y validación de una escala para medir síntomas maníacos. Escala para 
manía de la Universidad Nacional de Colombia (EMUN) Revista Colombiana de Psiquiatría. 2008;37:516-37.

20. Hambleton RK, Swaminathan H, Rogers HJ. Fundamentals of item response theory. Newbury Park: Sage Publications; 1991.

21. Linden WJ, Hambleton RK. Handbook of modern item response theory. New York: Springer; 1997.

22. Bond TG, Fox CM. Applying the Rasch model: Fundamental measurement in the human sciences. 2nd edition. Mahwah, N.J.: Lawrence Erlbaum Associates Publishers; 2007.

23. Wright BD, Linacre JM. Observations are always ordinal; measurements, however, must be interval. Arch Phys Med Rehabil. 1989;70:857-60.

24. Wright B. Reasonable mean-square fit values. Rasch Measurement Transactions. 2002;8:370.

25. Linacre JM. Optimizing rating scale category effectiveness. J Appl Meas. 2002;3:85-106.

26. DeMars C. Item response theory. Oxford: Oxford University Press; 2010.
27. Liu X, Boone WJ. Applications of Rasch measurement in science education. Maple Grove: JAM Press; 2006.

28. Cassidy F, Murry E, Forest K, Carroll BJ. Signs and symptoms of mania in pure and mixed episodes. J Affect Disord. 1998;50:187-201.

29. American Psychiatric Association. Diagnostic criteria from DSM-IV-TR. Washington, D.C.: American Psychiatric Association; 2000.

30. Rangel O, Sánchez R. Estudio de la estructura latente del síndrome maníaco mediante técnicas de escalamiento multidimensional. Revista Colombiana de Psiquiatría. 2010;39:240-50.

31. Cassidy F, Ahearn E, Carroll BJ. Concordance of selfrated and observer-rated dysphoric symptoms in mania. $\mathrm{J}$ Affect Disord. 2009;114:294-8.

32. Cassidy F, Carroll BJ. Frequencies of signs and symptoms in mixed and pure episodes of mania: Implications for the study of manic episodes. Prog Neuropsychopharmacol Biol Psychiatry. 2001;25:659-65. 